\title{
Wall teichoic acid is a pathogen-associated molecular pattern of Staphylococcus aureus that is recognized by langerin (CD207) on skin Langerhans cells
}

Rob van Dalen ${ }^{1}$, Matevž Rumpret ${ }^{1}$, Felix F. Fuchsberger ${ }^{1,2}$, Nienke H. van Teijlingen ${ }^{3}$, Jonas Hanske $^{2}$, Christoph Rademacher ${ }^{2}$, Theunis B.H. Geijtenbeek ${ }^{3}$, Jos A.G. van Strijp ${ }^{1}$, Christopher Weidenmaier ${ }^{4,5}$, Andreas Peschel $^{4,5}$ and Nina M. van Sorge ${ }^{1 *}$

${ }^{1}$ Medical Microbiology, University Medical Center Utrecht, Utrecht University; Heidelberglaan 100, 3584 CX Utrecht, The Netherlands

${ }^{2}$ Department of Biomolecular Systems, Max Planck Institute of Colloids and Interfaces; Am Mühlenberg 1, 14424, Potsdam, Germany

${ }^{3}$ Department of Experimental Immunology, Academic Medical Center, University of Amsterdam; Meibergdreef 9, 1105 AZ Amsterdam, The Netherlands

${ }^{4}$ Interfaculty Institute of Microbiology and Infection Medicine, University of Tübingen; Auf der Morgenstelle 28, 72076 Tübingen, Germany

${ }^{5}$ German Center for Infection Research (DZIF), Partnersite Tübingen; Tübingen, Germany

*Correspondence: nsorge3@umcutrecht.nl 


\section{Summary}

Staphylococcus aureus is a major cause of skin and soft tissue infections and aggravator of the inflammatory skin disease atopic dermatitis (AD). Langerhans cells (LCs) initiate a Th17 response upon exposure to $S$. aureus, which contributes to host defense but also to $A D$ pathogenesis. However, the molecular mechanisms underlying the unique pro-inflammatory capacities of S. aureus remain unclear. We demonstrate that human LCs directly interact with S. aureus through the pattern-recognition receptor langerin (CD207), which specifically recognizes the conserved $\beta-N$-acetylglucosamine (GIcNAc) modifications of wall teichoic acid (WTA) that are not expressed by other staphylococcal species. The WTA glycoprofile strongly influences the production of Th1- and Th17-polarizing cytokines by LCs. Specifically, $\beta$-GIcNAc activates LCs, whereas co-decoration of WTA with $\alpha$-GICNAc through the enzyme TarM, uniformly present in the AD-associated CC1 lineage, attenuates LC immune activation. Our findings provide important mechanistic insights into the role of $S$. aureus in inflammatory skin disease.

\section{Keywords}

Langerhans cell, langerin, CD207, Staphylococcus aureus, wall teichoic acid, glycosylation, atopic dermatitis, pathogen-associated molecular pattern 


\section{Introduction}

Staphylococus aureus is a common colonizer of the skin and nasal cavities but is also a major cause of skin disease. S. aureus-associated skin and soft tissue infections (SSTIs) are an important cause of disease and hospitalization and account for over 10 million cases each year in the US alone (Hersh et al., 2008). S. aureus is also an aggravator of the inflammatory skin disease atopic dermatitis (AD, eczema), which affects up to $20 \%$ of children and $3 \%$ of adults worldwide (Nutten, 2015). AD-affected skin is characterized by a disturbed composition of the microbiota with low species diversity and dominance of S. aureus (Totte et al., 2016). In particular, S. aureus strains of the CC1 lineage are overrepresented in AD isolates and were proposed to have particular yet-unidentified features that enable colonization of AD skin (Geoghegan et al., 2017).

When the skin barrier is breached by pathogens such as $S$. aureus a coordinated immune response is initiated. This response involves both resident and recruited immune cells as well as secreted antimicrobial factors. Important resident immune cells of the skin epidermis are Langerhans cells (LCs). These cells fulfill a gatekeeping function by sensing evolutionary conserved pathogen-associated molecular patterns (PAMPs) on invading pathogens through pattern recognition receptors (PRRs). In response, LCs can phagocytose invaders and initiate adaptive immune responses by activating skin-resident immune memory cells or naïve immune cells in the lymph nodes (Kissenpfennig et al., 2005, Seneschal et al., 2012). LCs are equipped with a diverse set of PRRs, including the LC-specific C-type lectin receptor (CLR) langerin (CD207) (Valladeau et al., 2000).

Wall teichoic acid (WTA) is an evolutionarily conserved and dominant component of the $S$. aureus cell wall. It plays an important role in nasal colonization, S. aureus-induced endocarditis, beta-lactam resistance and phage-mediated horizontal gene transfer (Winstel et al., 2015, Weidenmaier et al., 2005, Brown et al., 2012, Xia et al., 2011, Winstel et al., 2013). In the majority of S. aureus lineages, WTA is composed of a glycopolymer backbone of 20 - 40 ribitol-phosphate (RboP) subunits modified by $N$-acetylglucosamine (GlcNAc) and 
$D$-alanine residues. The GIcNAc modifications can be either $\alpha$-O-linked or $\beta$-O-linked to the anomeric C4 of the RboP subunits by the glycosyltransferases TarM and TarS, respectively (Xia et al., 2010, Brown et al., 2012). Overall, several S. aureus WTA glycoprofiles can be discriminated: WTA $\beta$-GIcNAcylation is conserved in almost all S. aureus strains, whereas WTA $\alpha$-GlcNAcylation is only present in about one third of the $S$. aureus isolates, and a small selection of isolates have no WTA glycosylation at all (Li et al., 2015, Winstel et al., 2015). In addition, WTA of S. aureus lineage ST395 is composed of a glycerol-phosphate (GroP) backbone modified by $\mathrm{N}$-acetylgalactosamine (GalNAc) instead of the common RboPGlcNAc WTA (Winstel et al., 2013).

WTA glycosylation is an important determinant in host-pathogen interactions through both innate and adaptive immune recognition. For example, opsonizing antibodies interact with WTA GIcNAc to initiate complement deposition and subsequent phagocytic uptake of $S$. aureus (Kurokawa et al., 2013). Innate immune component interacting with WTA include the scavenger receptor SREC-1, which is important for attachment of S. aureus to the nasal epithelium, and mannose-binding lectin, which initiates deposition of complement C4 on S. aureus (Baur et al., 2014, Park et al., 2010). The interactions with these innate receptors are not influenced by the anomeric configuration of WTA GIcNAc.

S. aureus can enter the epidermis through a damaged stratum corneum, which is an important predisposing determinant for AD (Nakatsuji et al., 2016). Epicutaneous exposure of LCs to S. aureus initiates Th17 responses, which contribute to host defense but also to AD pathogenesis (Igyártó et al., 2011, Kobayashi et al., 2015). Previous studies indeed show that S. aureus-exposed LCs induce T cell proliferation (van der Aar et al., 2013). However, the molecular interaction between LCs and S. aureus and the functional consequences have received little attention. Therefore, the aim of this study was to investigate the interaction between S. aureus and human LCs at a molecular and functional level. 
We demonstrate an important role of the PRR langerin in sensing the $S$. aureus cell wall component WTA. Langerin specifically recognizes $\beta$-GICNAC modifications on $S$. aureus WTA, which contributes to LC activation and production of Th1- and Th17-polarizing cytokines. The selectivity of langerin for $\beta$-GIcNAc also explains the lack of binding to other non-AD associated staphylococcal species. Interestingly, simultaneous decoration of WTA with $\alpha$-GICNAc impairs langerin interaction and attenuates the functional response of LCs, implying that $S$. aureus can modulate immune detection and subsequent inflammation in the epidermis. In conclusion, we identify WTA as a pathogen-associated molecular pattern of $S$. aureus, which is recognized by langerin on LCs. 


\section{Results}

\section{Langerin is a receptor for S. aureus on human LCs}

To determine whether LCs and S. aureus interact directly, we incubated primary LCs isolated from human skin with GFP-expressing S. aureus. LCs from four different donors bound S. aureus in a dose-dependent manner (Figure 1A). The levels at which the interaction was saturated varied between the donors from approximately $40 \%$ (donor 1 ) to $80 \%$ (donor 3 ) of S. aureus-positive LCs. To investigate the nature of interacting receptors on LCs, we preincubated LCs with mannan, a ligand for many CLRs. Depending on the bacteria-to-cell ratio, S. aureus binding was reduced by $35-70 \%$ compared to non-blocking conditions in all donors (Figure $1 \mathrm{~A}$ ). Similarly, the interaction was inhibited by approximately $35 \%$ by pre-incubation of the LCs with the monosaccharide GIcNAc. As langerin is a mannan- and GlcNAc-specific CLR that is exclusively expressed on LCs, we investigated whether langerin would be involved in interaction with S. aureus. Indeed, pre-incubation with an anti-langerin blocking antibody reduced binding of (spa and sbi-deficient) S. aureus in donors 3 and 4 by 25-50\% compared to control, depending on the infective dose (Figure 1A). To confirm involvement of langerin in the interaction between S. aureus and LCs, we introduced langerin in THP1 cells. Transduction of langerin, but not of empty vector (EV), conferred S. aureus binding to THP-1 cells, which could be completely inhibited by addition of mannan or anti-langerin blocking antibody (Figure 1B). Although it was previously demonstrated that S. aureus-exposed LCs initiate T cell proliferation (van der Aar et al., 2013), the functional response of LCs was not assessed. Therefore, we incubated MUTZ-3-derived LCs (muLCs), a well-established cell model for human LCs (Masterson et al., 2002, Santegoets et al., 2006), with S. aureus and measured muLC activation through expression of co-stimulatory molecules and cytokine production after 24 hours. Indeed, muLCs upregulated expression of co-stimulatory molecules CD80 and CD86 and produced significant amounts of IL-8 and IL-12p70 in a dose-dependent response to S. aureus (Figure 1C). Together, these data demonstrate that LCs respond to S. aureus and that langerin is an important innate receptor for $S$. aureus on human LCs. 


\section{Langerin recognizes $S$. aureus in a tarS-dependent manner through the conserved WTA $\beta$-GIcNAc epitope}

To further investigate langerin interaction with staphylococci, we tested binding of a FITClabeled trimeric construct of the extracellular domain of human langerin (langerin-FITC) to a broader collection of S. aureus strains (18 strains from 11 different clonal complexes (CCs)) as well as several coagulase-negative staphylococci (CoNS) (Figure 2A). Langerin-FITC bound to 16 out of $18(89 \%)$ tested S. aureus strains but to none of the tested CoNS species, indicating that langerin interacts with a ligand that is specific for and highly conserved in $S$. aureus. The three tested S. aureus strains that showed no or low-level binding of langerinFITC (ED133, Lowenstein and PS187; Figure 2A), differ from the other tested S. aureus strains in the structural composition of WTA. ED133 and Lowenstein completely lack WTA GlcNAcylation, whereas PS187 belongs to the ST395 lineage that expresses GroP-GalNAc WTA (Li et al., 2015, Lee et al., 2015, Winstel et al., 2013). Given the high density of WTA on the S. aureus surface and apparent correlation between langerin interaction and WTA structure, we hypothesized that WTA GICNAC modifications are likely candidates for the interaction with langerin.

To test this, we assessed binding of langerin-FITC to a panel of S. aureus knockout strains, which lack glycosyltransferases TarM and TarS required to modify WTA with $\alpha$-GIcNAc and $\beta$-GIcNAc, respectively. Loss of both glycosyltransferases ( $\triangle$ tarMS) reduced langerin-FITC binding to $S$. aureus to background levels (Figures 2B and S1BC), demonstrating that WTA GIcNAc is the target for langerin. To investigate whether langerin specifically recognized either $\alpha$-GICNAc or $\beta$-GICNAc, we tested the individual TarM and TarS knockout strains as well as $\triangle$ tarMS complemented with either tarM or tarS on an expression plasmid ( $\triangle \operatorname{tarMS}$ ptarM and $\Delta$ tarMS ptarS). Langerin-FITC only bound to $S$. aureus strains that express $\beta$ GlcNAc, whereas a-GlcNAc was dispensable for binding (Figures 2B and S1BC). Similarly, langerin-FITC binding to S. aureus strains 82086 and PS66, which are naturally deficient for WTA a-GlcNAc, was completely abrogated in isogenic $\Delta$ tarS strains (Figure 2C). These 
results show that langerin interacts with $S$. aureus in a tarS-dependent manner and provide the first demonstration of an anomeric-specific interaction of a human innate receptor with a Gram-positive surface polysaccharide.

Although a-GIcNAc is not the target of langerin, its presence or absence influences the level of langerin-FITC binding: mutant strains lacking tarM ( $\Delta \operatorname{tarM}$ and $\Delta \operatorname{tarMS}$ ptarS) showed significantly increased binding compared to wild-type (Figures 2B and S1BC). Enhanced binding is likely due to loss of shielding by a-GICNAc, since chemical analysis of the WTA composition by Kurokawa et al. suggests that $\triangle \operatorname{tar} M$ WTA does not have increased $\beta$ GlcNAcylation (Kurokawa et al., 2013).

Since S. aureus expresses many human-specific factors (Koymans et al., 2016), we also investigated the interaction with murine langerin-FITC, which has $76 \%$ identity with the human langerin-FITC construct (Hanske et al., 2017). Binding of murine langerin-FITC to S. aureus was detectable, however binding levels were 10 to 100 -fold lower than human langerin (Figure S1A), suggesting the langerin-S. aureus interaction displays a certain degree of species-specificity.

Altogether, these data show that human langerin interacts with $S$. aureus in a tarSdependent manner through the conserved $\beta$-GIcNAc WTA modification and that the level of interaction is influenced by the simultaneous modification with a-GIcNAc.

\section{S. aureus-induced production of Th1/Th17 polarizing cytokines by LCs is influenced}

\section{by the WTA glycoprofile}

Given the importance of langerin for interaction between S. aureus and LCs, we investigated whether distinct WTA GICNAC glycoprofiles influenced the LC response at the level of costimulatory molecules and cytokine expression. Stimulation of muLCs for $24 \mathrm{~h}$ with $\mathrm{y}$ irradiated wildtype S. aureus (WT) induced upregulation of activation markers CD80, CD83 and CD86 (Figure 3A). Stimulation with $\beta$-GICNAc deficient S. aureus ( $\Delta \operatorname{tar} S$ ) reduced expression of these markers compared to WT, whereas stimulation with $\alpha$-GIcNAc deficient S. aureus $(\Delta \operatorname{tar} M)$ enhanced expression. MuLCs secreted significant levels of IL-6, IL-8 IL- 
12p70, IL-23p19 and TNFa (Figure 3B), but not anti-inflammatory cytokine IL-10 (not shown), in response to S. aureus. Especially IL-8, IL-12p70 and IL-23p19 were highly produced. Similar to the activation markers, stimulation of muLCs with S. aureus $\Delta \operatorname{tar} S$ reduced IL-6, IL8, IL-12p70, IL-23p19 and TNFa secretion compared to WT, whereas stimulation with S. aureus $\Delta$ tarM significantly enhanced secretion of these cytokines (Figure 3B). Interestingly, the level of activation matched the interaction levels of recombinant langerin-FITC to $S$. aureus WT, $\Delta \operatorname{tarM}$ and $\Delta \operatorname{tarS}$ strains (Figure 2B). These data suggest that the previously described Th17-polarizing response initiated by LCs in response to S. aureus is strongly influenced by detection of WTA $\beta$-GIcNAc. Moreover, the presence of WTA $\alpha$-GIcNAc attenuates the LC response to $S$. aureus. 


\section{Discussion}

Despite the emerging role of LCs in S. aureus-mediated skin inflammation, the molecular interaction between LCs and S. aureus has not been clarified. We identify WTA as a PAMP of $S$. aureus, of which the $\beta$-GIcNAc modifications are recognized by langerin on LCs. This interaction contributes to LC activation and production of Th1- and Th17-polarizing cytokines. Importantly, the extend of LC activation is strongly influenced by the WTA glycoprofile and suggest the possibility for immune evasion. Although several innate receptors have been described for $S$. aureus WTA, langerin is the first human innate receptor to discriminate between the $\alpha$-GIcNAc and $\beta-$ GIcNAc modifications.

Similar to lipopolysaccharide (LPS) of Gram-negative bacteria, WTA is an abundant evolutionarily conserved feature on the surface of Gram-positive bacteria and essential for maintenance of cell wall architecture and bacterial physiology. Therefore, it is advantageous for the host to recognize such structures in a timely manner through PRRs that are strategically localized at mucosal surfaces. In the case of $S$. aureus, a common colonizer of skin but also a major cause of skin disease, recognition of $S$. aureus WTA by langerin on epidermal LCs could be key to maintaining skin homeostasis. Since bacterial physiology is severely affected without WTA (Weidenmaier and Lee, 2016), bacteria have evolved other ways to regulate WTA expression and composition. First of all, WTA density depends on expression of $\operatorname{tarH}$, which encodes the energy generating component of the WTA ABC transporter TarGH. Importantly, the amount of WTA correlates to the ability of S. aureus to induce skin abscess formation (Wanner et al., 2017). Second, studies on lytic podophage infection of S. aureus suggest careful regulation of WTA GlcNAcylation (Li et al., 2015). In support of this, in silico genome analysis identified tarM as part of the GraRS regulon (Falord et al., 2011), which is known to control resistance to cationic antimicrobials, oxidative stress and growth at high temperatures. Whether and how conditions during skin colonization affect WTA density or GIcNAcylation remains to be determined and will require the development of analytical tools that can accurately and immediately analyze the WTA glycoprofile. 
We show that LCs respond to $S$. aureus with production of a Th1/Th17-polarizing cytokine profile. These data corroborate finding by others, who have demonstrated that LCs internalize S. aureus and subsequently polarize T cells towards Th17 (Igyártó et al., 2011, Kobayashi et al., 2015, van der Aar et al., 2013, Iwamoto et al., 2017). We now elucidate that detection of S. aureus WTA $\beta$-GIcNAc is of critical importance for the induced response. LCs and langerin were previously implicated in host defense against various other pathogens. LCs internalize and break down HIV-1 viral particles in a langerin-dependent manner to prevent infection of deeper layers of the mucosa (de Witte et al., 2007, Ribeiro et al., 2016). Langerin has also been identified as a major receptor for fungal pathogens on LCs through recognition of mannose and beta-glucan structures (de Jong et al., 2010). The Gramnegative bacterium Yersinia pestis is the only other bacterium known to interact with langerin through its LPS core oligosaccharide (Yang et al., 2015). We hereby identify S. aureus as a new langerin-interacting pathogen.

As is the case for many S. aureus virulence factors, we observe a certain degree of species specificity for the interaction between langerin and WTA (Koymans et al., 2016). Despite the high homology between human and mouse langerin (66\% identity), the binding levels of mouse langerin were approximately 50 -fold lower compared to human langerin. This corroborates previous studies that reported differences in ligand specificity of these orthologs (Hanske et al., 2017). It has become increasingly clear that mouse and human skin immunology can be very different, varying from altered receptor specificities to unique cell types and associated functions. This limits the translation of data obtained from mouse studies to humans. The different ligand specificities and thereby S. aureus WTA binding potential of human and mouse langerin are yet another example of this.

S. aureus is a driver of AD disease progression, which is mediated by LCs (Kobayashi et al., 2015). Interestingly, CD207, the gene encoding for langerin, was identified in genome-wide association studies as an AD susceptibility locus (Paternoster et al., 2015, Cai et al., 2017). Curiously, polymorphisms in a putative enhancer region of CD207, which are suggested to 
enhance expression of langerin, were protective for $A D$. Our data now functionally link langerin to S. aureus. One might hypothesize that enhanced langerin expression results in enhanced bacterial recognition, which helps to eradicate $S$. aureus and prevents the development of chronic inflammation as observed in AD. However, an alternative explanation could be that $S$. aureus exploits langerin interaction to intentionally elicit inflammation to perturb the skin barrier and release nutrients. Since $S$. aureus is largely resistant to host defenses but most of the other commensals are not, this could explain the strong association between $S$. aureus and $A D$ and the described driver function of $S$. aureus in $A D$ disease progression. This suggests that langerin could play an important role in sensing and regulating skin microbiota homeostasis.

Although S. aureus is associated with AD in general, the CC1 lineage is overrepresented in isolates from $A D$ skin (Geoghegan et al., 2017). This lineage was suggested to have particular yet-unidentified features that enable colonization of and proliferation in AD skin. Interestingly, all CC1 strains are tarM-positive (Winstel et al., 2014). Therefore, CC1 strains have the potential to regulate their WTA glycoprofile, balancing the inflammatory status of the skin and gaining an advantage to colonize AD skin. Therefore, our data provide molecular insight into the association between $A D$ and $S$. aureus from two different angles: on the immunological side we show how langerin and LCs are involved in the immune response to S. aureus, while on the microbiological side the involvement of langerin could explain the association of $S$. aureus but not CoNS species with $A D$, and possibly also the overrepresentation of tarM-bearing CC1 strains in AD.

In conclusion, we identify WTA as a PAMP on S. aureus and pinpoint langerin as a molecular trigger for S. aureus-induced skin immune responses. Our findings may have implications for our deeper understanding of the association of $S$. aureus with skin inflammation and can help in the development of new targeted treatment strategies for the treatment of $S$. aureusassociated skin and soft tissue infections and inflammatory skin diseases. 


\section{Acknowledgements}

The authors would like to thank the Multiplex Core Facility (UMC Utrecht, The Netherlands) for performing the Luminex assay, David Gerlach, Guoqing Xia and Volker Winstel (University of Tübingen, Germany) for advice and communication regarding the shipment of strains, Samantha van der Beek, Eline van Yperen and Małgorzata Mnich (UMC Utrecht, The Netherlands) for technical assistance, and Tanja de Gruijl (VU University Medical Center, Amsterdam, The Netherlands) for the protocol detailing MUTZ-3 culture and differentiation.

This work was supported by a VIDI grant (91713303) from the Dutch Scientific Organization (NWO) to N.M.v.S. A.P. is supported by German Research Council (DFG) grants TRR34, TRR156 and the German Center for Infection Research (DZIF). J.H. thanks the DFG Collaborative Research Centre 765 "Multivalency" for a fellowship. C.R. thanks the DFG for funding (RA1944/2-1).

\section{Author Contributions}

Conceptualization, R.v.D. and N.M.v.S.;

Methodology, R.v.D., M.R. and N.M.v.S.;

Investigation, R.v.D. and M.R.;

Resources, F.F.F., N.H.v.T., J.H., C.R., T.B.H.G., C.W. and A.P.;

Writing - Original Draft, R.v.D. and N.M.v.S.;

Writing - Review \& Editing, R.v.D., J.A.G.v.S., C.W., A.P. and N.M.v.S.;

Visualization, R.v.D.;

Supervision, J.A.G.v.S. and N.M.v.S.;

Funding Acquisition, N.M.v.S.

\section{Declaration of Interests}

The authors declare no competing interests. 


\section{References}

Baur, S., Rautenberg, M., Faulstich, M., Grau, T., Severin, Y., Unger, C., Hoffmann, W. H., Rudel, T., Autenrieth, I. B. and Weidenmaier, C. (2014). A nasal epithelial receptor for Staphylococcus aureus WTA governs adhesion to epithelial cells and modulates nasal colonization. PLoS Pathog, 10, e1004089.

Ben Zakour, N. L., Bannoehr, J., Van Den Broek, A. H., Thoday, K. L. and Fitzgerald, J. R. (2011). Complete genome sequence of the canine pathogen Staphylococcus pseudintermedius. J Bacteriol, 193, 2363-4.

Brown, S., Xia, G., Luhachack, L. G., Campbell, J., Meredith, T. C., Chen, C., Winstel, V., Gekeler, C., Irazoqui, J. E., Peschel, A., et al. (2012). Methicillin resistance in Staphylococcus aureus requires glycosylated wall teichoic acids. Proc Natl Acad Sci U S A, 109, 18909-14.

Cai, X. Y., Zheng, X. D., Fang, L., Zhou, F. S., Sheng, Y. J., Wu, Y. Y., Yu, C. X., Zhu, J. and Xiao, F. L. (2017). A variant on chromosome 2p13.3 is associated with atopic dermatitis in Chinese Han population. Gene, 628, 281-285.

Centers for Disease Control and Prevention (1999). Four pediatric deaths from communityacquired methicillin-resistant Staphylococcus aureus - Minnesota and North Dakota, 1997-1999. MMWR Morb Mortal Wkly Rep, 48, 707-10.

Chassain, B., Lemee, L., Didi, J., Thiberge, J. M., Brisse, S., Pons, J. L. and Pestel-Caron, M. (2012). Multilocus sequence typing analysis of Staphylococcus lugdunensis implies a clonal population structure. J Clin Microbiol, 50, 3003-9.

De Jong, M. A., Vriend, L. E., Theelen, B., Taylor, M. E., Fluitsma, D., Boekhout, T. and Geijtenbeek, T. B. (2010). C-type lectin Langerin is a beta-glucan receptor on human Langerhans cells that recognizes opportunistic and pathogenic fungi. Mol Immunol, 47, 1216-25.

De Witte, L., Nabatov, A., Pion, M., Fluitsma, D., De Jong, M. A., De Gruijl, T., Piguet, V., Van Kooyk, Y. and Geijtenbeek, T. B. (2007). Langerin is a natural barrier to HIV-1 transmission by Langerhans cells. Nat Med, 13, 367-71.

Falord, M., Mader, U., Hiron, A., Debarbouille, M. and Msadek, T. (2011). Investigation of the Staphylococcus aureus GraSR regulon reveals novel links to virulence, stress response and cell wall signal transduction pathways. PLoS One, 6, e21323.

Geoghegan, J. A., Irvine, A. D. and Foster, T. J. (2017). Staphylococcus aureus and Atopic Dermatitis: A Complex and Evolving Relationship. Trends Microbiol.

Guinane, C. M., Ben Zakour, N. L., Tormo-Mas, M. A., Weinert, L. A., Lowder, B. V., Cartwright, R. A., Smyth, D. S., Smyth, C. J., Lindsay, J. A., Gould, K. A., et al. (2010). Evolutionary genomics of Staphylococcus aureus reveals insights into the origin and molecular basis of ruminant host adaptation. Genome Biol Evol, 2, 454-66.

Hanske, J., Schulze, J., Aretz, J., Mcbride, R., Loll, B., Schmidt, H., Knirel, Y., Rabsch, W., Wahl, M. C., Paulson, J. C., et al. (2017). Bacterial Polysaccharide Specificity of the Pattern Recognition Receptor Langerin Is Highly Species-dependent. J Biol Chem, 292, 862-871.

Hersh, A. L., Chambers, H. F., Maselli, J. H. and Gonzales, R. (2008). National trends in ambulatory visits and antibiotic prescribing for skin and soft-tissue infections. Arch Intern Med, 168, 1585-91.

Igyártó, B. Z., Haley, K., Ortner, D., Bobr, A., Gerami-Nejad, M., Edelson, B. T., Zurawski, S. M., Malissen, B., Zurawski, G., Berman, J., et al. (2011). Skin-resident murine dendritic cell subsets promote distinct and opposing antigen-specific $\mathrm{T}$ helper cell responses. Immunity, 35, 260-72.

Iwamoto, K., Moriwaki, M., Niitsu, Y., Saino, M., Takahagi, S., Hisatsune, J., Sugai, M. and Hide, M. (2017). Staphylococcus aureus from atopic dermatitis skin alters cytokine production triggered by monocyte-derived Langerhans cell. J Dermatol Sci.

Kissenpfennig, A., Henri, S., Dubois, B., Laplace-Builhe, C., Perrin, P., Romani, N., Tripp, C. H., Douillard, P., Leserman, L., Kaiserlian, D., et al. (2005). Dynamics and function of 
Langerhans cells in vivo: dermal dendritic cells colonize lymph node areas distinct from slower migrating Langerhans cells. Immunity, 22, 643-54.

Kobayashi, T., Glatz, M., Horiuchi, K., Kawasaki, H., Akiyama, H., Kaplan, D. H., Kong, H. H., Amagai, M. and Nagao, K. (2015). Dysbiosis and Staphylococcus aureus Colonization Drives Inflammation in Atopic Dermatitis. Immunity, 42, 756-66.

Koymans, K. J., Vrieling, M., Gorham, R. D., Jr. and Van Strijp, J. A. (2016). Staphylococcal Immune Evasion Proteins: Structure, Function, and Host Adaptation. Curr Top Microbiol Immunol.

Kreiswirth, B. N., Lofdahl, S., Betley, M. J., O'reilly, M., Schlievert, P. M., Bergdoll, M. S. and Novick, R. P. (1983). The toxic shock syndrome exotoxin structural gene is not detectably transmitted by a prophage. Nature, 305, 709-12.

Kurokawa, K., Jung, D. J., An, J. H., Fuchs, K., Jeon, Y. J., Kim, N. H., Li, X., Tateishi, K., Park, J. A., Xia, G., et al. (2013). Glycoepitopes of staphylococcal wall teichoic acid govern complement-mediated opsonophagocytosis via human serum antibody and mannose-binding lectin. J Biol Chem, 288, 30956-68.

Lee, J. H., Kim, N. H., Winstel, V., Kurokawa, K., Larsen, J., An, J. H., Khan, A., Seong, M. Y., Lee, M. J., Andersen, P. S., et al. (2015). Surface Glycopolymers Are Crucial for In Vitro Anti-Wall Teichoic Acid IgG-Mediated Complement Activation and Opsonophagocytosis of Staphylococcus aureus. Infect Immun, 83, 4247-55.

Li, X., Gerlach, D., Du, X., Larsen, J., Stegger, M., Kuhner, P., Peschel, A., Xia, G. and Winstel, V. (2015). An accessory wall teichoic acid glycosyltransferase protects Staphylococcus aureus from the lytic activity of Podoviridae. Sci Rep, 5, 17219.

Mack, D., Siemssen, N. and Laufs, R. (1992). Parallel induction by glucose of adherence and a polysaccharide antigen specific for plastic-adherent Staphylococcus epidermidis: evidence for functional relation to intercellular adhesion. Infect Immun, 60, 2048-57.

Masterson, A. J., Sombroek, C. C., De Gruijl, T. D., Graus, Y. M., Van Der Vliet, H. J., Lougheed, S. M., Van Den Eertwegh, A. J., Pinedo, H. M. and Scheper, R. J. (2002). MUTZ-3, a human cell line model for the cytokine-induced differentiation of dendritic cells from CD34+ precursors. Blood, 100, 701-3.

Monk, I. R., Shah, I. M., Xu, M., Tan, M. W. and Foster, T. J. (2012). Transforming the untransformable: application of direct transformation to manipulate genetically Staphylococcus aureus and Staphylococcus epidermidis. MBio, 3.

Mwangi, M. M., Wu, S. W., Zhou, Y., Sieradzki, K., De Lencastre, H., Richardson, P., Bruce, D., Rubin, E., Myers, E., Siggia, E. D., et al. (2007). Tracking the in vivo evolution of multidrug resistance in Staphylococcus aureus by whole-genome sequencing. Proc Natl Acad Sci U S A, 104, 9451-6.

Nakatsuji, T., Chen, T. H., Two, A. M., Chun, K. A., Narala, S., Geha, R. S., Hata, T. R. and Gallo, R. L. (2016). Staphylococcus aureus Exploits Epidermal Barrier Defects in Atopic Dermatitis to Trigger Cytokine Expression. J Invest Dermatol.

Nutten, S. (2015). Atopic dermatitis: global epidemiology and risk factors. Ann Nutr Metab, 66 Suppl 1, 8-16.

Pang, Y. Y., Schwartz, J., Thoendel, M., Ackermann, L. W., Horswill, A. R. and Nauseef, W. M. (2010). agr-Dependent interactions of Staphylococcus aureus USA300 with human polymorphonuclear neutrophils. J Innate Immun, 2, 546-59.

Park, K. H., Kurokawa, K., Zheng, L., Jung, D. J., Tateishi, K., Jin, J. O., Ha, N. C., Kang, H. J., Matsushita, M., Kwak, J. Y., et al. (2010). Human serum mannose-binding lectin senses wall teichoic acid Glycopolymer of Staphylococcus aureus, which is restricted in infancy. J Biol Chem, 285, 27167-75.

Paternoster, L., Standl, M., Waage, J., Baurecht, H., Hotze, M., Strachan, D. P., Curtin, J. A., Bonnelykke, K., Tian, C., Takahashi, A., et al. (2015). Multi-ancestry genome-wide association study of 21,000 cases and 95,000 controls identifies new risk loci for atopic dermatitis. Nat Genet, 47, 1449-56.

Ribeiro, C. M., Sarrami-Forooshani, R., Setiawan, L. C., Zijlstra-Willems, E. M., Van Hamme, J. L., Tigchelaar, W., Van Der Wel, N. N., Kootstra, N. A., Gringhuis, S. I. and 
Geijtenbeek, T. B. (2016). Receptor usage dictates HIV-1 restriction by human TRIM5alpha in dendritic cell subsets. Nature, 540, 448-452.

Rosenstein, R., Nerz, C., Biswas, L., Resch, A., Raddatz, G., Schuster, S. C. and Gotz, F. (2009). Genome analysis of the meat starter culture bacterium Staphylococcus carnosus TM300. Appl Environ Microbiol, 75, 811-22.

Santegoets, S. J., Masterson, A. J., Van Der Sluis, P. C., Lougheed, S. M., Fluitsma, D. M., Van Den Eertwegh, A. J., Pinedo, H. M., Scheper, R. J. and De Gruijl, T. D. (2006). A CD34(+) human cell line model of myeloid dendritic cell differentiation: evidence for a CD14(+)CD11b(+) Langerhans cell precursor. J Leukoc Biol, 80, 1337-44.

Schenk, S. and Laddaga, R. A. (1992). Improved method for electroporation of Staphylococcus aureus. FEMS Microbiol Lett, 73, 133-8.

Seneschal, J., Clark, R. A., Gehad, A., Baecher-Allan, C. M. and Kupper, T. S. (2012). Human epidermal Langerhans cells maintain immune homeostasis in skin by activating skin resident regulatory T cells. Immunity, 36, 873-84.

Sibbald, M. J., Winter, T., Van Der Kooi-Pol, M. M., Buist, G., Tsompanidou, E., Bosma, T., Schafer, T., Ohlsen, K., Hecker, M., Antelmann, H., et al. (2010). Synthetic effects of secG and secY2 mutations on exoproteome biogenesis in Staphylococcus aureus. J Bacteriol, 192, 3788-800.

Totte, J. E., Van Der Feltz, W. T., Hennekam, M., Van Belkum, A., Van Zuuren, E. J. and Pasmans, S. G. (2016). Prevalence and odds of Staphylococcus aureus carriage in atopic dermatitis: a systematic review and meta-analysis. Br J Dermatol.

Valladeau, J., Ravel, O., Dezutter-Dambuyant, C., Moore, K., Kleijmeer, M., Liu, Y., DuvertFrances, V., Vincent, C., Schmitt, D., Davoust, J., et al. (2000). Langerin, a novel Ctype lectin specific to Langerhans cells, is an endocytic receptor that induces the formation of Birbeck granules. Immunity, 12, 71-81.

Van De Weijer, M. L., Van Muijlwijk, G. H., Visser, L. J., Costa, A. I., Wiertz, E. J. and Lebbink, R. J. (2016). The E3 Ubiquitin Ligase TMEM129 Is a Tri-Spanning Transmembrane Protein. Viruses, 8.

Van Der Aar, A. M., Picavet, D. I., Muller, F. J., De Boer, L., Van Capel, T. M., Zaat, S. A., Bos, J. D., Janssen, H., George, T. C., Kapsenberg, M. L., et al. (2013). Langerhans cells favor skin flora tolerance through limited presentation of bacterial antigens and induction of regulatory T cells. J Invest Dermatol, 133, 1240-9.

Wanner, S., Schade, J., Keinhorster, D., Weller, N., George, S. E., Kull, L., Bauer, J., Grau, T., Winstel, V., Stoy, H., et al. (2017). Wall teichoic acids mediate increased virulence in Staphylococcus aureus. Nat Microbiol, 2, 16257.

Weidenmaier, C. and Lee, J. C. (2016). Structure and Function of Surface Polysaccharides of Staphylococcus aureus. Curr Top Microbiol Immunol.

Weidenmaier, C., Peschel, A., Xiong, Y. Q., Kristian, S. A., Dietz, K., Yeaman, M. R. and Bayer, A. S. (2005). Lack of wall teichoic acids in Staphylococcus aureus leads to reduced interactions with endothelial cells and to attenuated virulence in a rabbit model of endocarditis. J Infect Dis, 191, 1771-7.

Winstel, V., Kuhner, P., Salomon, F., Larsen, J., Skov, R., Hoffmann, W., Peschel, A. and Weidenmaier, C. (2015). Wall Teichoic Acid Glycosylation Governs Staphylococcus aureus Nasal Colonization. MBio, 6, e00632.

Winstel, V., Liang, C., Sanchez-Carballo, P., Steglich, M., Munar, M., Broker, B. M., Penades, J. R., Nubel, U., Holst, O., Dandekar, T., et al. (2013). Wall teichoic acid structure governs horizontal gene transfer between major bacterial pathogens. Nat Commun, 4, 2345.

Winstel, V., Xia, G. and Peschel, A. (2014). Pathways and roles of wall teichoic acid glycosylation in Staphylococcus aureus. Int J Med Microbiol, 304, 215-21.

Xia, G., Corrigan, R. M., Winstel, V., Goerke, C., Grundling, A. and Peschel, A. (2011). Wall teichoic Acid-dependent adsorption of staphylococcal siphovirus and myovirus. J Bacteriol, 193, 4006-9. 
Xia, G., Maier, L., Sanchez-Carballo, P., Li, M., Otto, M., Holst, O. and Peschel, A. (2010). Glycosylation of wall teichoic acid in Staphylococcus aureus by TarM. J Biol Chem, 285, 13405-15.

Yang, K., Park, C. G., Cheong, C., Bulgheresi, S., Zhang, S., Zhang, P., He, Y., Jiang, L., Huang, H., Ding, H., et al. (2015). Host Langerin (CD207) is a receptor for Yersinia pestis phagocytosis and promotes dissemination. Immunol Cell Biol, 93, 815-24. 


\section{Figure Legends}

\section{Figure 1. Langerin is a receptor for S. aureus on human LCs}

(A) Binding of S. aureus to isolated primary human LCs. LCs from donors 1 and 2 were incubated with GFP-expressing S. aureus Newman and LCs from donors 3 and 4 with GFPexpressing S. aureus Newman $\Delta s p a \Delta s b i$ and binding was assessed by flow cytometry. The interaction was blocked by addition of mannan $(10 \mu \mathrm{g} / \mathrm{ml})$, GlcNAc $(50 \mathrm{mM})$ or anti-langerin blocking antibody (20 $\mu \mathrm{g} / \mathrm{ml}$, donors 3 and 4 only).

(B) Binding of S. aureus to THP1-langerin cells. Human langerin-transduced or empty vector (EV)-transduced THP1 cells were incubated with GFP-expressing S. aureus Newman $\Delta s p a \Delta s b i$. The interaction was blocked by addition of mannan $(10 \mu \mathrm{g} / \mathrm{ml})$ or anti-langerin blocking antibody $(20 \mu \mathrm{g} / \mathrm{ml})$. Within each ratio, THP1-langerin was compared to the other conditions.

(C) Expression of co-stimulatory molecules CD80 and CD86 and production of cytokines IL-8 and IL12p70 by muLCs after 24h incubation with y-irradiated S. aureus USA300.

All data are represented as geometric mean fluorescent intensity or mean concentration + SEM from three independent experiments. ${ }^{*} p<0.05$, ${ }^{\star *} p<0.01,{ }^{* \star} p<0.001$.

Figure 2. Langerin recognizes $S$. aureus in a tarS-dependent manner through the conserved WTA $\beta$-GIcNAc epitope

Binding of recombinant human langerin-FITC to (A) 18 wild-type S. aureus strains (11 different clonal complexes, indicated above the bars) and a selection of coagulase-negative staphylococcal species (CoNS); (B) S. aureus USA300 wild-type (WT) and WTA biosynthesis mutants $\Delta \operatorname{tarMS}, \Delta \operatorname{tar} M, \Delta \operatorname{tar} S, \Delta \operatorname{tar} M S$ ptarM and $\Delta \operatorname{tar} M S$ ptarS; and (C) two representative S. aureus strains (82086 and PS66) that naturally lack tarM and their isogenic $\Delta$ tarS mutants. All strains were grown to mid-exponential phase and incubated with $5 \mu \mathrm{g} / \mathrm{ml}$ langerin-FITC or no langerin-FITC (background). Binding was assessed by flow cytometry. Data are represented as geometric mean fluorescence intensity + SEM from three independent experiments. ${ }^{\star} p<0.05,{ }^{* \star} p<0.01,{ }^{\star \star *} p<0.001$. See also Figure S1. 
Figure 3. S. aureus-induced production of Th1/Th17 polarizing cytokines by LCs is influenced by the WTA glycoprofile

(A) Expression of co-stimulatory molecules CD80 and CD86 and maturation marker CD83 and (B) production of cytokines IL-6, IL-8, IL12p70, IL23p19 and TNFa by muLCs. muLCs were incubated $24 \mathrm{~h}$ with $\mathrm{Y}$-irradiated S. aureus USA300 wild-type (WT), $\Delta \operatorname{tar} M$ and $\Delta \operatorname{tar} S$. muLCs stimulated with WT S. aureus were compared to the unstimulated control, whereas muLCs stimulated with $\Delta \operatorname{tarM}$ and $\Delta$ tarS were compared to their respective WT controls within the same ratio. Data are represented as geometric mean fluorescence intensity or mean concentration + SEM from three independent experiments. ${ }^{\star} p<0.05$, ${ }^{\star *} p<0.01$, ${ }^{\star \star *} p<$ 0.001. 


\section{STAR Methods}

\section{CONTACT FOR REAGENT AND RESOURCE SHARING}

Further information and requests for resources and reagents should be directed to and will be fulfilled by the Lead Contact, Dr. Nina M. van Sorge (nsorge3@umcutrecht.nl).

\section{EXPERIMENTAL MODEL AND SUBJECT DETAILS}

\section{Ethics statement}

Human skin tissue was collected from otherwise healthy donors undergoing corrective breast or abdominal surgery. This study, including the tissue harvesting procedures, were approved by the Medical Ethics Review Committee of the Academic Medical Center.

\section{Bacterial culture}

S. aureus, S. capitis, S. carnosus, S. epidermidis, S. lugdunensis, S. pseudintermedius, S. saprophyticus and S. simulans strains were grown overnight at $37^{\circ} \mathrm{C}$ with agitation in $5 \mathrm{ml}$ Todd-Hewitt broth (THB; Oxoid). For S. aureus strains that were plasmid complemented THB was supplemented with $10 \mu \mathrm{g} / \mathrm{ml}$ chloramphenicol (Sigma Aldrich). A fresh $5 \mathrm{ml}$ THB culture was inoculated by $150 \mu$ overnight culture and grown to an optical density at $600 \mathrm{~nm}$ $\left(\mathrm{OD}_{600 \mathrm{~nm}}\right)$ of 0.4 for $S$. capitis and to $\mathrm{OD}_{600 \mathrm{~nm}}$ 0.6-0.7 for all other bacteria, which corresponds to mid-exponential growth phase.

\section{Cell culture and differentiation}

MUTZ-3 cells (human, male; ACC-295, DSMZ) were cultured in a 12-well tissue culture plates (Corning) at a density of $0.5-1.0 \times 10^{6}$ cells $/ \mathrm{ml}$ in MEM-alpha (Gibco) with $20 \%$ fetal bovine serum (FBS, Hyclone, GE Healthcare), 1\% GlutaMAX (Gibco), 10\% conditioned supernatant from renal carcinoma cell line 5637 (ACC-35, DSMZ), $100 \mathrm{U} / \mathrm{ml}$ penicillin and $100 \mu \mathrm{g} / \mathrm{ml}$ streptomycin (Gibco) at $37^{\circ} \mathrm{C}$ with $5 \% \quad \mathrm{CO}_{2}$. We obtained MUTZ-3 derived Langerhans cells (muLCs) by differentiation of MUTZ-3 cells for 10 days in $100 \mathrm{ng} / \mathrm{ml}$ Granulocyte-Macrophage Colony Stimulating Factor (GM-CSF, GenWay Biotech), 10 ng/ml Transforming Growth Factor-beta (TGF $\beta$; R\&D Systems) and $2.5 \mathrm{ng} / \mathrm{ml}$ Tumor Necrosis 
Factor-alpha (TNFa; R\&D Systems) as described previously (Masterson et al., 2002, Santegoets et al., 2006). The phenotype of differentiated muLCs was verified by surface staining of CD34 (clone 581, BD Biosciences), CD1a (clone HI149, BD Biosciences) and CD207 (clone DCGM4, Beckman Coulter) using the respective antibodies and analysis by flow cytometry (FACSVerse, BD Biosciences). Data were analyzed using FlowJo 10 (FlowJo, LLC).

THP1 cells (human, male; TIB-202, ATCC) transduced with a lentiviral langerin construct or empty vector (EV) were cultured in RPMI (Lonza) supplemented with 5\% FBS (Biowest), 1\% GlutaMAX $100 \mathrm{U} / \mathrm{ml}$ penicillin and $100 \mu \mathrm{g} / \mathrm{ml}$ streptomycin (Gibco) at $37^{\circ} \mathrm{C}$ with $5 \% \mathrm{CO}_{2}$.

\section{METHOD DETAILS}

\section{Isolation of primary human Langerhans cells}

Human Langerhans cells were isolated as described previously (Ribeiro et al., 2016). In short, skin grafts were obtained using a dermatome (Zimmer) and incubated in medium supplemented with Dispase II (1 U/ml, Roche Diagnostics) after which epidermal sheets were separated from the dermis and cultured for three days. After incubation, migrated LCs were harvested and further purified using a Ficoll gradient (Axis-shield). Isolated LCs were routinely 90\% pure (CD1a+ Langerin+) and were frozen in Iscoves Modified Dulbeccos's Medium (IMDM, Thermo Fischer Scientific) supplemented with 20\% FBS and 10\% DMSO. Before use, LCs were thawed by drop-wise addition of cold IMDM with 10\% FBS, washed twice and incubated in IMDM with FBS for 2 hours at $37^{\circ} \mathrm{C}$ with $5 \% \mathrm{CO}_{2}$ to recover.

\section{Creation of GFP-expression S. aureus}

To create GFP-expressing bacteria, S. aureus Newman wildtype and S. aureus Newman $\Delta s p a \Delta s b i$ were transformed as described previously with pCM29, which encodes superfolded green fluorescent protein (SGFP) driven by the sarAP1 promoter (Pang et al., 2010, Schenk and Laddaga, 1992). In short, competent S. aureus were electroporated with pCM29 isolated from E. coli DC10B with a Gene Pulser II (BioRad; 100 Ohm, 25uF, 2.5kV). 
After recovery, bacteria were selected on $\mathrm{TH}$ agar supplemented with $10 \mu \mathrm{g} / \mathrm{ml}$ chloramphenicol. A single colony was grown in THB with $10 \mu \mathrm{g} / \mathrm{ml}$ chloramphenicol under the usual growth conditions. Bacterial expression of GFP was verified by confocal laser scanning microscopy (Leica SP5, Leica).

\section{Gamma-irradiation of S. aureus}

Gamma-irradiated stocks of $S$. aureus strains were made by harvesting cultures in midexponential growth phase by centrifugation (4,000 rpm, $8 \mathrm{~min})$, which were concentrated 10x in phosphate-buffered saline (PBS; Lonza) with $17 \%$ glycerol (VWR), frozen at $-70^{\circ} \mathrm{C}$ and exposed to $10 \mathrm{kGy}$ of $\mathrm{Y}$-radiation (Synergy Health, Ede, The Netherlands). Loss of viability of S. aureus was verified by plating of the irradiated bacteria. A non-irradiated aliquot that underwent the same freezing procedure was used to determine the concentration of colony forming units (CFU) of the irradiated stocks.

\section{Lentiviral transduction}

A TrueORF sequence-validated cDNA clone of human CD207 (OriGene Technologies) was amplified by PCR using Phusion polymerase (Thermo Fisher) and primers hLangerin-Fw and hLangerin-FLAG-Rv (IDT). The PCR amplicon was cloned in a BIC-PGK-Zeo-T2amAmetrine;EF1A construct by Gibson assembly (NEB) according to the manufacturer's instructions. The langerin-encoding vector and an empty vector (EV) control were introduced into THP1 cells by lentiviral transduction, as described by Van de Weijer et al. (van de Weijer et al., 2016). In short, lentivirus was produced by HEK293T cells (CRL-3216, ATCC) in 24well plates using standard lentiviral production protocols and third-generation packaging vectors. After 3-4 days the supernatant containing the viral particles was harvested and stored at $-70^{\circ} \mathrm{C}$ to kill any remaining cells. Approximately 50,000 THP1 cells were transduced by spin infection $\left(1000 \times \mathrm{xg}, 2 \mathrm{~h}, 33^{\circ} \mathrm{C}\right)$ using $100 \mu \mathrm{l}$ supernatant supplemented with 8 $\mu \mathrm{g} / \mathrm{ml}$ polybrene (Santa Cruz Biotechnology). Complete medium was added after centrifugation and cells were selected three days post-infection by $100 \mu \mathrm{g} / \mathrm{ml}$ zeocin (Gibco). 
Cellular expression of langerin was verified by antibody staining of langerin (DCGM4, Beckman Coulter) and measured using flow cytometry.

\section{Bacterial binding assays}

To test binding of bacteria to cells, $10^{5} \mathrm{LCs}$, THP1-EV or THP1-langerin were incubated with GFP-expressing S. aureus Newman or GFP-expressing S. aureus Newman $\Delta s p a \Delta s b i$ at bacteria-to-cell ratios from 1 to 8 in TSM buffer (2.4 g/L Tris (Roche), $8.77 \mathrm{~g} / \mathrm{L} \mathrm{NaCl}$ (Sigma Aldrich), $294 \mathrm{mg} / \mathrm{L} \mathrm{CaCl}_{2} \cdot 2 \mathrm{H} 2 \mathrm{O}$ (Merck), $294 \mathrm{mg} / \mathrm{L} \mathrm{MgCl}_{2} \cdot 6 \mathrm{H} 2 \mathrm{O}$ (Merck), pH=7.4) with 0.1\% bovine serum albumin (BSA; Merck) for 30 minutes at $4^{\circ} \mathrm{C}$. Binding was blocked by 15 minutes pre-incubation with $10 \mu \mathrm{g} / \mathrm{ml}$ mannan (Sigma Aldrich), $50 \mathrm{mM}$ GlcNAc (Serva) or 20 $\mu \mathrm{g} / \mathrm{ml}$ anti-langerin blocking antibody (10E2, Sony Biotechnology). Cells were washed once with TSM 1\% BSA, fixed in 1\% formaldehyde (Brunschwig Chemie) and measured by flow cytometry. We assessed bacterial binding by LCs or THP1 cells as the percentage of GFPpositive cells.

\section{Production of recombinant langerin extracellular domains}

The extracellular domains of truncated human langerin (residues 148-328) and mouse langerin (residues 150-331) were recombinantly expressed from codon-optimized constructs containing a C-terminal TEV cleavage site followed by a Strep-tag II cloned into pUC19 and pET30a (EMD Millipore) expression vectors as described previously (Hanske et al., 2017). Recombinant human and murine ECDs were insolubly expressed in E. coli BL21(DE3), solubilized in $6 \mathrm{M}$ guanidinium hydrochloride in $100 \mathrm{mM}$ Tris $(\mathrm{pH}$ 8) with $1 \mathrm{mM}$ DTT, refolded by dialyisis against Tris-buffered saline $(\mathrm{pH}$ 7.5) containing $10 \mathrm{mM} \mathrm{CaCl}$ and purified via mannan-coupled sepharose beads (Sigma Aldrich). Bound protein was eluted with Trisbuffered saline $(\mathrm{pH} 7.5)$ containing 5 mM EDTA. Protein concentrations were determined by A280 nm using the calculated molar extinction coefficients of $56,170 \mathrm{M}^{-1} \mathrm{~cm}^{-1}$ for the human langerin ECD and $56,170 \mathrm{M}^{-1} \mathrm{~cm}^{-1}$ for the murine ECD. The proteins were fluorescently labeled with fluorescein isothiocyanate (FITC, Thermo Fisher) by adding slowly $100 \mu \mathrm{L}$ of the dye solution ( $1 \mathrm{mg} / \mathrm{ml}$ in DMSO) to $2 \mathrm{ml}$ of a $2 \mathrm{mg} / \mathrm{ml}$ protein solution in HEPES-buffered 
saline (pH 7.2) containing $20 \mathrm{mM}$ D-mannose (Sigma Aldrich) and $5 \mathrm{mM} \mathrm{CaCl}_{2}$. After stirring for $90 \mathrm{~min}$ at room temperature, the reaction was quenched by addition of $50 \mathrm{mM}$ ethanolamine ( $\mathrm{pH}$ 8.5, Sigma Aldrich). Unreacted dye molecules were removed by buffer exchange using a Zeba spin column (Thermo Fisher) and active protein was purified over mannan affinity column as described above. All chemicals used for the production of recombinant langerin extracellular domains were obtained from Carl Roth if not indicated otherwise.

\section{Langerin binding assay}

Bacteria in mid-exponential growth phase were harvested by centrifugation $(4,000 \mathrm{rpm}, 8$ minutes) and resuspended at $\mathrm{OD}_{600 \mathrm{~nm}} 0.4$ in TSM buffer with $0.1 \%$ BSA. Bacteria were incubated with $1-50 \mu \mathrm{g} / \mathrm{ml}$ recombinant langerin-FITC (human or mouse) for 30 minutes at $37^{\circ} \mathrm{C}$ with agitation, washed once with TSM $1 \%$ BSA, fixed in $1 \%$ formaldehyde and analyzed by flow cytometry. We determined langerin-FITC binding of the bacteria using the geometric mean signal in the FITC channel.

\section{muLC stimulation}

We stimulated $5 \times 10^{4}$ muLCs with S. aureus USA300 WT, USA300 $\Delta$ tarM or USA300 $\Delta$ tarS at bacteria-to-cell ratios of $0,1,10$ and 50 in IMDM with 10\% FBS. After 24 hours, supernatants were collected by centrifugation (300xg, $10 \mathrm{~min}, 4^{\circ} \mathrm{C}$ ) and stored at $-150^{\circ} \mathrm{C}$ until further analysis, and cells were washed once in PBS 0.1\% BSA. Expression levels of the activation and maturation markers CD80, CD83 and CD86 were determined by flow cytometry using antibody clones 2D10 (CD80), HB15e (CD83) and IT2.2 (CD86) (Sony Biotechnology) and their corresponding isotype controls (BD Biosciences).

\section{Cytokine assays}

The IL-8 and IL12p70 concentrations were initially determined by ELISA (Sanquin and Thermo Fisher, respectively) according to the manufacturer's instructions. Concentrations of 
IL-6, IL-8, IL-10, IL-12p70, IL-23p19 and TNFa were determined by Luminex xMAP assay (Luminex Corporation), performed by Multiplex Core Facility UMC Utrecht, The Netherlands.

\section{QUANTIFICATION AND STATISTICAL ANALYSIS}

We analyzed flow cytometry data using FlowJo 10 (FlowJo LLC). Statistical analyses were performed using Graphpad Prism 7.02 (GraphPad Software). We used unpaired two-tailed $t$ tests for comparisons between two groups and one-way ANOVAs with a common control group followed by Dunnett's test for comparisons between multiple groups. The human and mouse langerin concentration curves were tested using a two-way ANOVA followed by Tukey's test for multiple comparisons. Differences were considered significant at $p \leq 0.05$. 
Fig.1
A

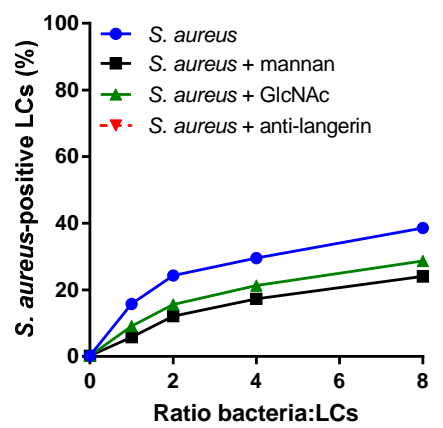

B

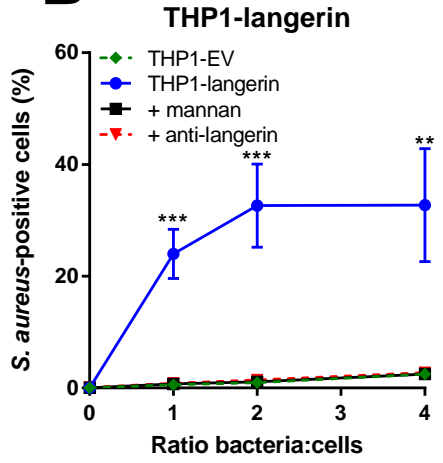

Donor 2

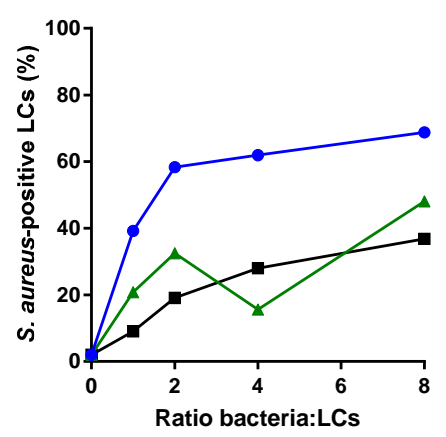

C

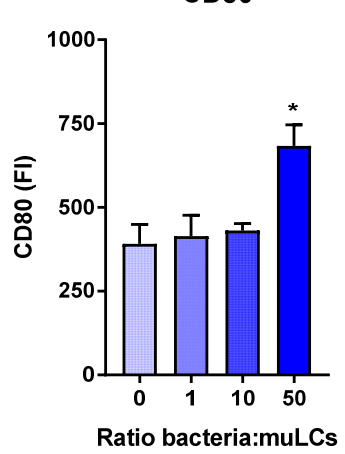

Donor 3

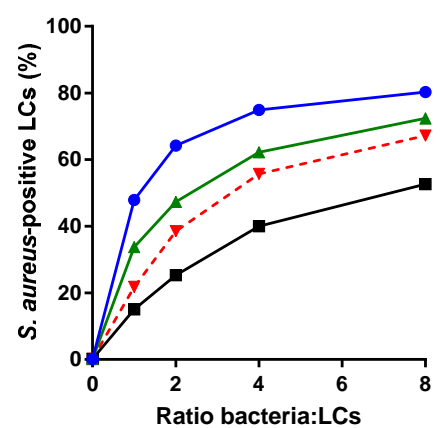

Donor 4
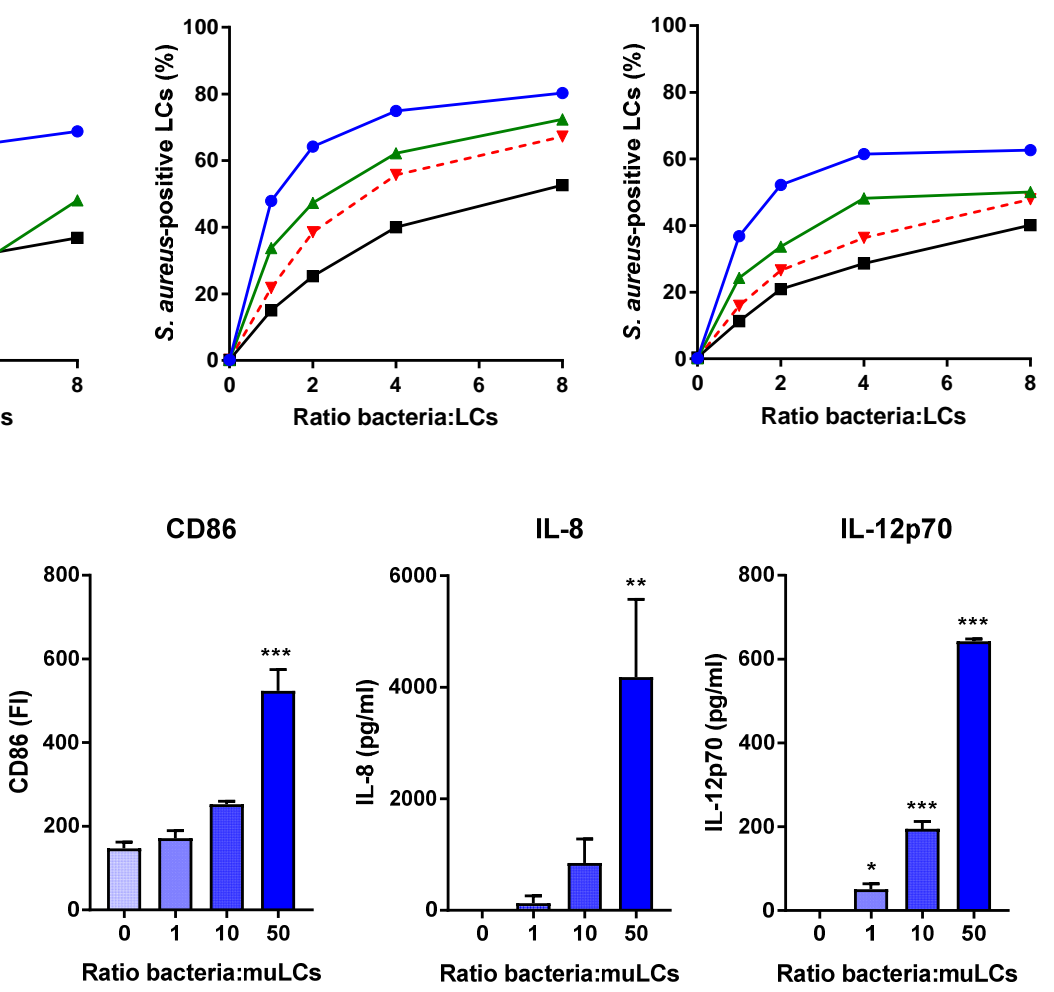

IL-8

IL-12p70

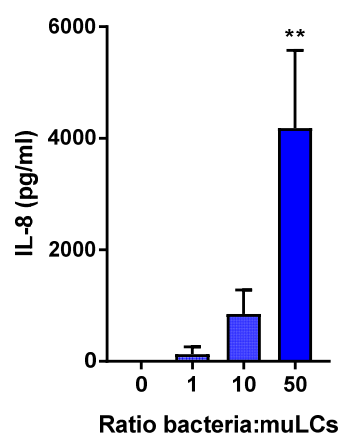

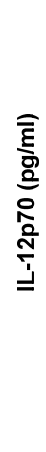




\section{Fig.2}

\section{A}

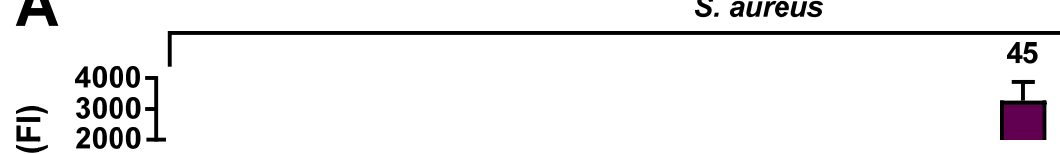

言 20001

ํㅗㅇำ

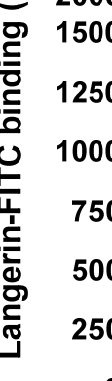

$\left.\begin{array}{r}1500 \\ 1250 \\ 750 \\ 500 \\ 250\end{array}\right]$

5
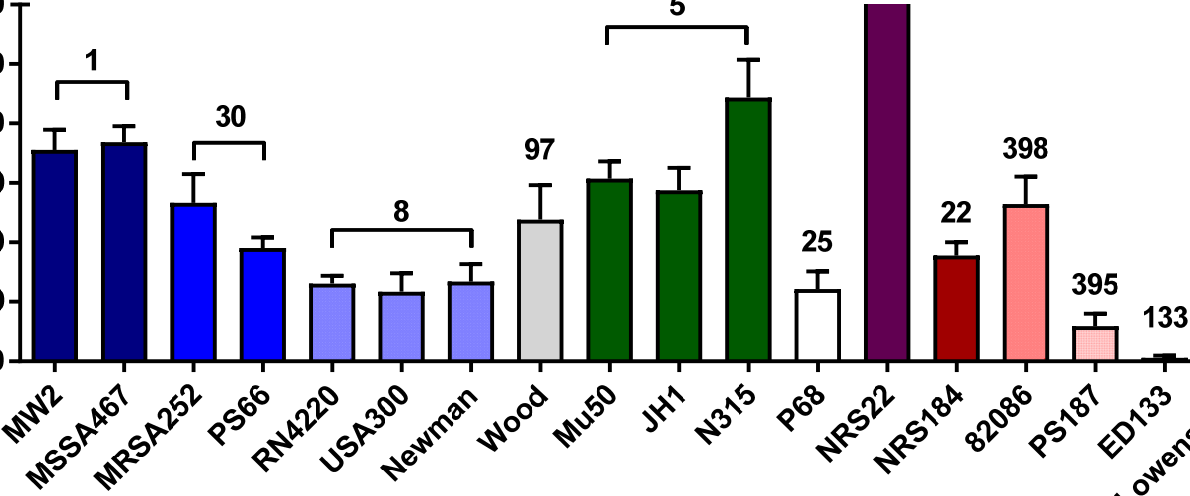

B

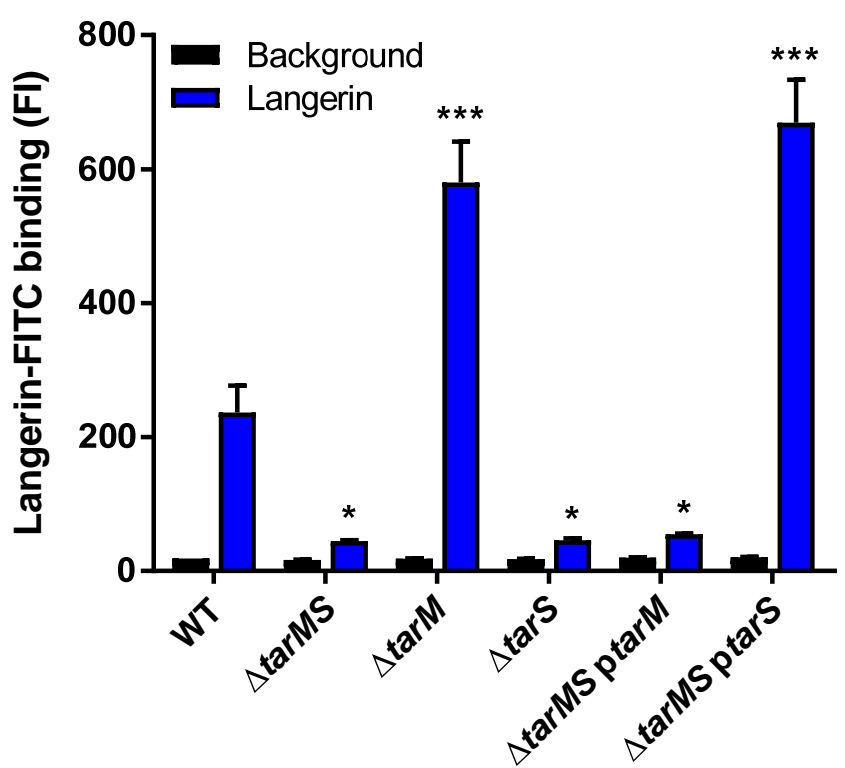

C

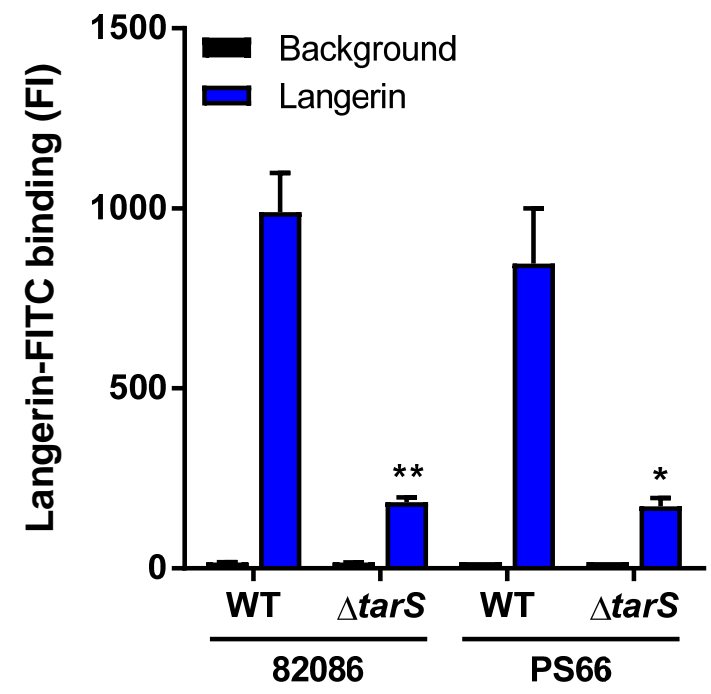


Fig.3

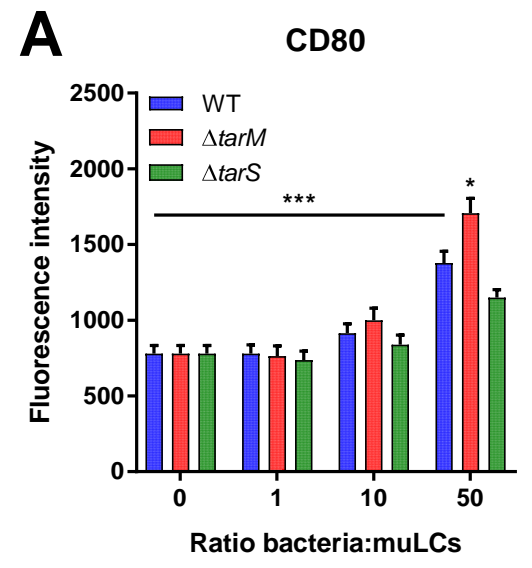

B

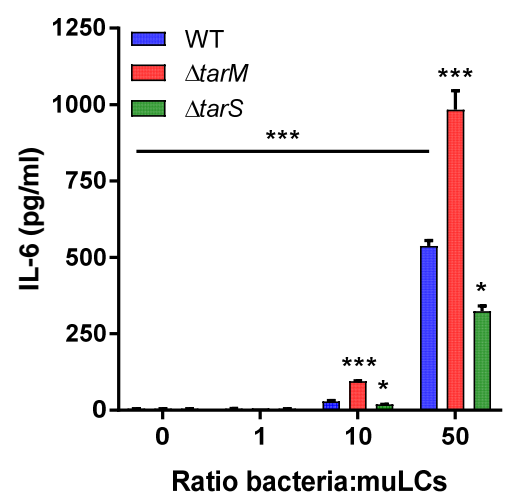

IL-23p19

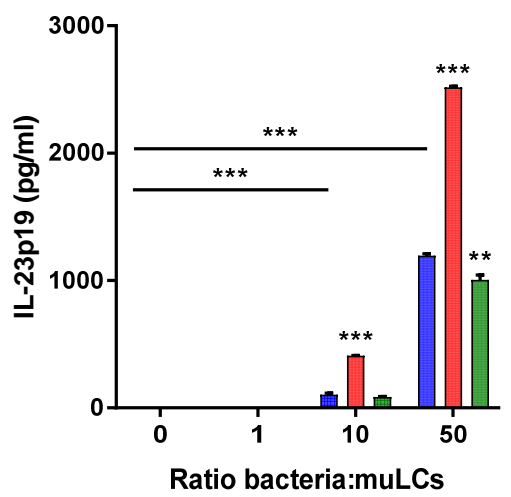

CD83

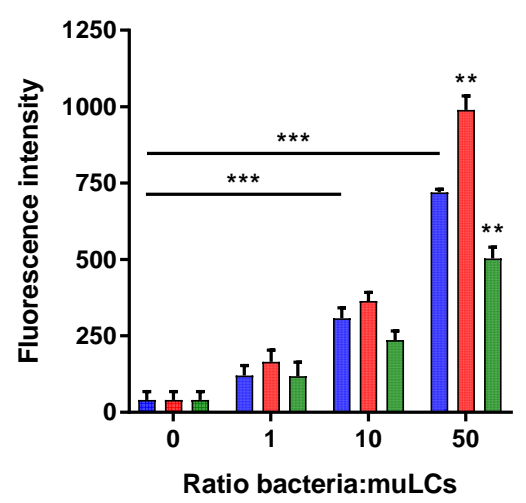

IL-8

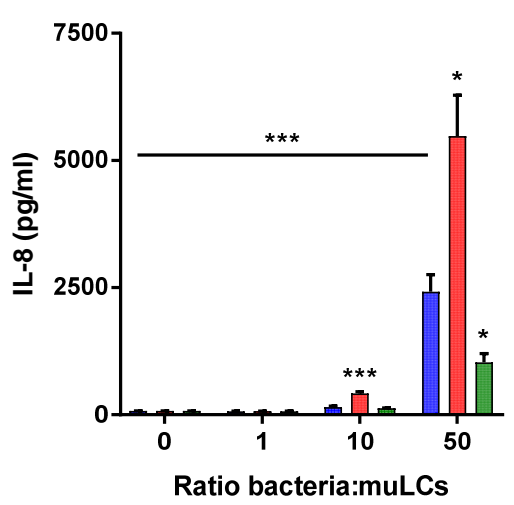

TNF $\alpha$

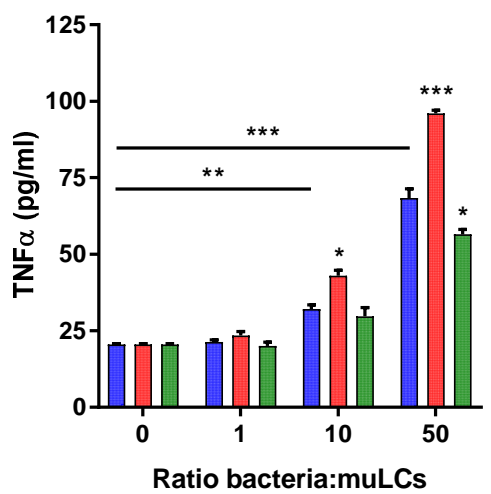

CD86

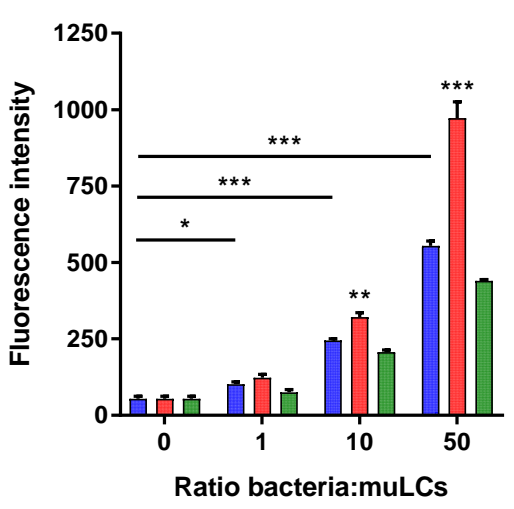

IL-12p70

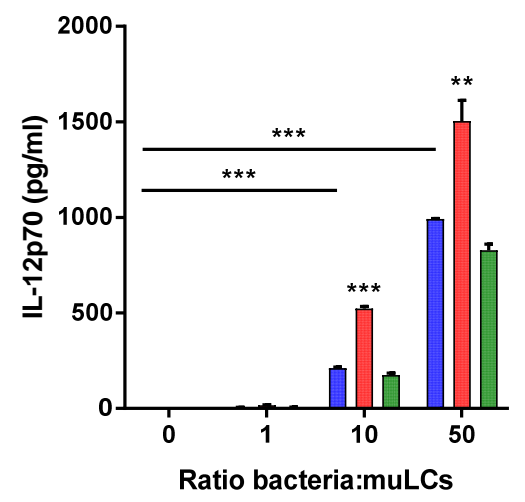

